\title{
An ISFET-based anion sensor for the potentiometric detection of organic acids in liquid chromatography
}

\author{
I. Poels ${ }^{\text {a }}$, R.B.M. Schasfoort ${ }^{\mathrm{b}}$, S. Picioreanu ${ }^{\mathrm{c}, 1}$, J. Frank ${ }^{\mathrm{c}}$, G.W.K. van Dedem ${ }^{\mathrm{c}}$, \\ A. van den Berg ${ }^{\mathrm{b}}$, L.J. Nagels ${ }^{\mathrm{a}, *}$ \\ a Department of Chemistry, University of Antwerpen (RUCA), Groenenborgerlaan 171, B-2020 Antwerp, Belgium \\ ${ }^{\mathrm{b}}$ MESA Research Institute, University of Twente, P.O. Box 217, 7500 AE Enschede, Netherlands \\ ${ }^{c}$ Kluyver Laboratory for Biotechnology, Delft University of Technology, Julianalaan 67, 2628 BC Delft, Netherlands
}

Received 14 February 2000; received in revised form 12 May 2000; accepted 15 May 2000

\begin{abstract}
An ion-selective field effect transistor (ISFET) was applied as a potentiometric detector in liquid chromatography (LC) for the determination of organic acids. The ISFET was prepared by coating the gate insulator of the encapsulated transistor with a poly(vinyl chloride) (PVC) matrix membrane containing methyltridodecylammoniumchloride, which enables the detection of organic anions. The ISFET was tested for its applicability as detector for carboxylic acids in ion-exchange and reversed-phase chromatography. Its analytical characteristics were compared to those of a coated-wire electrode (CWE) and of a conventional type of ion-selective electrode (ISE). (C) 2000 Elsevier Science S.A. All rights reserved.
\end{abstract}

Keywords: ISFET; Liquid chromatography; Potentiometric detection; Carboxylic acids

\section{Introduction}

In liquid chromatography (LC), several systems are used routinely for the determination of organic acids, including reversed-phase [1,2], ion-exchange [3], ion-exclusion [2,4] and ion-pair [5] chromatography. Also, capillary electrophoresis (CE) has proved to be a successful technique for the separation and quantification of shortchain carboxylic acids [6].

In this study two chromatographic separation methods, namely reversed-phase (RP) and ion-exchange were applied. UV- and conductivity detection are most commonly employed in combination with these systems. However, for carboxylic acids, which lack chromophoric groups, low UV detection is necessary. This implies a loss in sensitivity and the occurrence of matrix interferences, especially when complex samples are analysed. The sensitivity of

\footnotetext{
* Corresponding author. Fax: +32-3-2180233.

E-mail address: lnagels@ruca.ua.ac.be (L.J. Nagels).

${ }^{1}$ On leave from University "Politehnica" of Bucharest, Bucharest, Romania.
}

conductivity detection may be limited by a large background signal. Therefore, a suppressor system is usually used in combination with a conductivity detector to reduce eluent conductivity. Potentiometric detection can be an interesting alternative in these cases. It is a relatively simple and inexpensive detection method. A distinct advantage of potentiometric detectors is that their response is independent on the size of the electrode that makes them well suited for use in combination with micro-separation techniques such as CE. Still, potentiometric sensors are not yet routinely applied in LC or in CE. Only a limited number of research groups work on this subject. Simon et al. applied liquid-membrane-based potentiometric detection in LC [7] and in CE [8]. Chen et al. employed a metallic copper electrode [5,9] and a tungsten oxide electrode [10] for the LC determination of organic acids. In the work of Hauser et al., different classes of organic ions were detected in $\mathrm{CE}$, at a metallic copper electrode [11] and at coated-wire electrodes (CWEs) [12]. Isildak [13] recently used an all solid-state contact tubular PVC-matrix membrane electrode for the detection of anions in ionchromatography. In our laboratory we applied liquid-membrane electrodes $[14,15]$ and conducting polymer coated 
electrodes $[16,17]$ for the detection of organic acids in LC and CE.

Ion-selective field effect transistors (ISFETs) are mainly employed in static measurements, comparable with the conventional way of using ion-selective electrodes (ISEs). However, the use of ISFETs in flow-through and flow-injection analysis (FIA) systems has also been widely documented $[18,19]$. As far as we know, only one paper reports the use of an ISFET in chromatography [20], namely for the determination of alkali-metal cations in ion-chromatography. In the present study, we examined the possibility of using an ISFET-based anion sensor as a potentiometric detector for organic acids separated using two liquid chromatographic methods: ion-exchange and reversed-phase. The analytical characteristics of the ISFET were compared with those of a CWE and of a conventional type of ISE with internal reference solution.

\section{Experimental}

\subsection{Reagents}

All the chemicals used were of analytical reagent grade. Eluents were prepared daily and were filtered through a $0.2 \mu \mathrm{m}$ membrane filter and degassed with helium before use. The eluent used in ion-exchange chromatography was prepared by dilution of a $50 \%(\mathrm{w} / \mathrm{w}) \mathrm{NaOH}$ solution with low carbonate concentration, obtained from J.T. Baker. This eluent was kept under a helium atmosphere during the analysis to avoid carbonate contamination. Organic acids were purchased from Merck, UCB, Acros, CRB, Aldrich, Fluka, Sigma and LBC. Stock solutions of fumaric acid and of organic acid mixtures were prepared in deionised water. Dilutions of the stock solutions in the running eluent were made daily and were filtered $(0.2 \mu \mathrm{m})$ before injection.

\subsection{Ion-selective membranes}

The composition of the anion-selective membrane was $6 \%$ methyltridodecylammonium-chloride, $65 \% o$ nitrophenyl octyl ether and $29 \%$ poly(vinyl chloride) (PVC). $350 \mathrm{mg}$ of the membrane material was dissolved in $3.5 \mathrm{ml}$ tetrahydrofuran (THF) and this solution was used directly after mixing. All membrane components were obtained from Fluka (Buchs, Switzerland).

Membranes for conventional ISEs were prepared by pouring the membrane cocktail into a glass ring $(30 \mathrm{~mm}$ diameter), which was placed on a glass plate and dried at room temperature for at least $24 \mathrm{~h}$. After the membrane was peeled off from the glass plate, a disk membrane was cut out and glued onto a PVC tube ( $3 \mathrm{~mm}$ diameter). The other end of the PVC tube was connected to a disposable 5-ml syringe. A silver/silver chloride wire was used as an internal reference electrode. The internal filling solution consisted of $0.1 \mathrm{M} \mathrm{KCl}$ containing $10^{-3} \mathrm{M}$ tetrapentylammoniumchloride.

To prepare the CWEs, the membrane mixture was deposited directly onto a glassy carbon (GC) electrode. Before coating, the GC electrodes (3 $\mathrm{mm}$ diameter), mounted in plastic bodies, were polished with a $5 \mu \mathrm{m}$-grid polishing sheet and cleaned with water and ethanol. Three layers were deposited consecutively on the electrode by using a Pasteur pipette. The THF was allowed to evaporate at room temperature for at least $2 \mathrm{~h}$.

Ion-selective membranes for ISFETs were formed by casting three consecutive drops of the membrane cocktail onto the gate area by using a Pasteur pipette. The membrane was dried for at least $2 \mathrm{~h}$ at room temperature.

\subsection{ISFET}

The ISFET chips used in the present study were developed and produced at the MESA + Research Institute of the University of Twente (Enschede, The Netherlands). The chip had a total size of $3 \times 4 \mathrm{~mm}$. The ISFET was an n-channel depletion mode FET, having as gate insulator a layer of $\mathrm{SiO}_{2}$ (75 nm thick), covered by a layer of $\mathrm{Ta}_{2} \mathrm{O}_{5}$ of $125 \mathrm{~nm}$ thick. The gate area had a size of $15 \times 500 \mu \mathrm{m}$. For details of the fabrication sequence of the ISFET, see Ref. [21]. The ISFET chips were mounted onto a cylindrical plastic body and encapsulated, except for the gate region, using epoxy resin $\left(\mathrm{Hysol}^{\circledR}\right.$ epoxy obtained from Dexter Electronics Materials Division NY). The ISFET chips were placed in such a way that the gate region was in the centre of the plastic body.

\subsection{Apparatus}

The LC-system comprised a SP8810 isocratic pump (Spectra Physics, San Jose, CA) and a Valco injector (50 $\mu 1$ loop). For ion-exchange chromatography, a pellicular anion-exchange column (IonPac AS11, $250 \times 4 \mathrm{~mm}$ i.d., Dionex, Sunnyvale, CA) was used in series with a guard column (Dionex IonPac AG11, $50 \times 4$ mm i.d.). A RP-8 column (Merck Lichrospher RP-8; $5 \mu \mathrm{m}, 125 \times 4 \mathrm{~mm}$ i.d.) was used for reversed phase chromatography. For measurements, the ISFETs, CWEs and ISEs were placed in a home-made large-volume wall-jet type flow cell [22]. A schematic representation of the flow cell with mounted ISFET is given in Fig. 1. The column effluent was directed perpendicularly towards the ion-selective membrane. The distance from the LC tubing-outlet to the ion-selective membrane was $100 \mu \mathrm{m}$. An Orion 800500 Ross electrode was used as reference electrode. The response of the ISFETs was measured with a source and drain follower type of ISFET amplifier (Sprenkels Consultancy, Lelystad, The Netherlands) in a constant drain-current mode, with a constant drain-source potential $(0.5 \mathrm{~V})$. The output signal was recorded unamplified on a PC 1000 data acquisition system from Thermo Separation Products (San Jose, CA). 


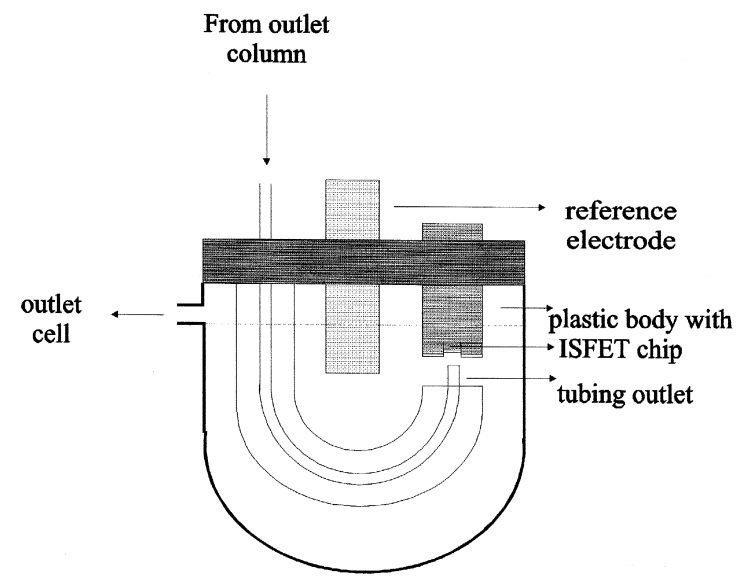

Fig. 1. Schematic representation of the flow cell with mounted ISFET.

In the case of the CWEs and ISEs, the membrane potential was measured against the reference electrode using a high impedance amplifier (internal resistance $10^{13} \Omega$, Knick, type $87 \mathrm{~F}$ ). The signals were amplified 10 times with a home-made amplifier and then recorded by the data acquisition system.

\section{Results and discussion}

The use of a potentiometric flow-through detector for $\mathrm{LC}$ requires the choice of a suitable eluent and an appropriate membrane coating material.

A suitable eluent is an eluent that permits both an efficient chromatographic separation and a sensitive detection. Both the analyte ion $i$, and the eluent ion $j$ have a contribution to the response of the potentiometric detectors. This response can be given by the Nikolskii-Eisenman equation:

$E=E \circ+\frac{R T}{z_{i} F} \ln \left(c_{i}+K_{i j}^{\mathrm{pot}} c_{j}^{\frac{z_{i}}{z_{j}}}\right)$

where $E \circ$ is a constant potential, $c_{i}$ and $z_{i}$ represent the concentration and the charge of the analyte ion, and $c_{j}$ and $z_{j}$ refer to the eluent ion. $K_{i j}^{\text {pot }}$ is the selectivity coefficient that describes the response of the sensor for the eluent ion $j$ versus the analyte ion $i$. A sensitive detection can only be obtained when the concentration of the eluent ion is relatively low and if it has a low $K_{i j}^{\text {pot }}$ value.

Membrane electrodes based on classical ion-exchangers such as quaternary ammonium compounds generally display the following selectivity series: $\mathrm{ClO}_{4}^{-}>\mathrm{SCN}^{-}>\mathrm{I}^{-}>$ $\mathrm{NO}_{3}^{-}>\mathrm{Br}^{-}>\mathrm{Cl}^{-}>\mathrm{HCO}_{3}^{-} \sim \mathrm{OAc}^{-} \sim \mathrm{SO}_{4}^{2-} \sim \mathrm{HPO}_{4}^{2-}$ [23]. This sequence is known as the Hofmeister series. It is dependent on the free enthalpy of hydration of these analyte anions. Therefore, the electrode is more selective for lipophilic anions. Potentiometric selectivity coefficients for the electrode used in this study were determined by the separate solution method and were published in Ref. [24].

\subsection{Ion-exchange chromatography}

In anion-exchange chromatography, the acids were separated in their anionic forms using a strongly alkaline $\mathrm{NaOH}$ solution as an eluent. In a recent study [24], it was shown that the coating material used in this work allows a sensitive detection of organic acids in this system. This is because the membrane material shows a high selectivity towards lipophilic anions (Hofmeister behaviour). Therefore, the electrode material is not very responsive to the
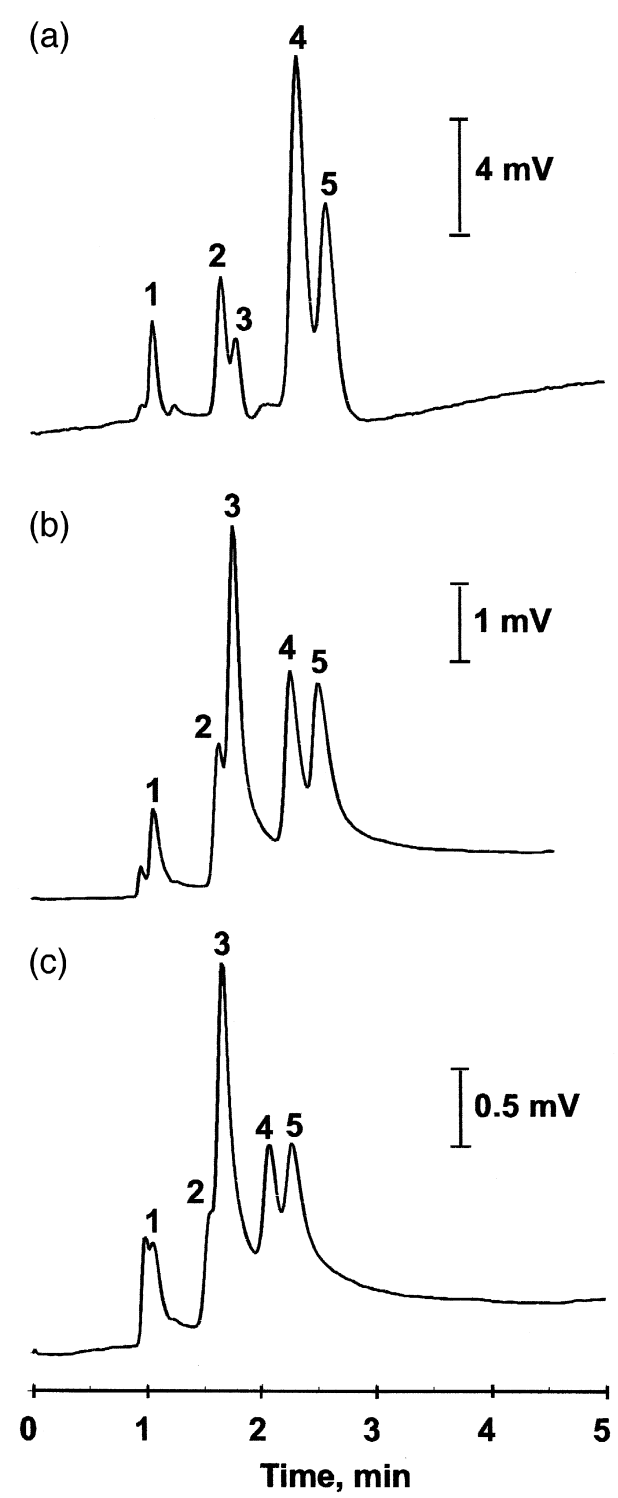

Fig. 2. Chromatograms of a mixture of four carboxylic acids obtained using three different potentiometric sensor types: (a) ISFET, (b) CWE and (c) ISE. Column: Dionex Ion Pac AS11, $250 \times 4 \mathrm{~mm}$ i.d. Flow-rate: $1.5 \mathrm{ml} \mathrm{min}{ }^{-1}$. Injection volume: $50 \mu 1$. Eluent: $8 \mathrm{mM} \mathrm{NaOH}$. Injected concentration: $10^{-4}$ M. Peak identification: (1) pyruvic acid, (2) malic acid, (3) system peak, (4) ketoglutaric acid and (5) fumaric acid. 
hydroxyl eluent anion, which is a very hydrophilic anion, but is highly responsive to the organic acid anions.

An $8 \mathrm{mM} \mathrm{NaOH}$ eluent $(\mathrm{pH}=12.1)$ was used to separate a mixture of four carboxylic acids of analytical interest. It contained pyruvic, malic, $\alpha$-ketoglutaric and fumaric acid. The chromatograms obtained with the ISFET, CWE and ISE are shown in Fig. 2.

In the case of fumaric acid, the detection limit, determined for a signal-to-noise ratio of 3 , was $2 \times 10^{-6} \mathrm{M}$ for all the three sensors. This corresponds to an injected amount of 100 pmol and is comparable to the value obtained with suppressed conductivity detection [25].

Calibration curves for fumaric acid were measured with the ISFET, CWE and ISE, showing peak height (in $\mathrm{mV}$ ) versus the logarithm of injected concentrations. They are presented in Fig. 3. A logarithmic relationship was observed for injected concentrations higher than $5 \times 10^{-5} \mathrm{M}$. At lower concentrations the curves showed a linear dependence, as predicted by a model described earlier [15]. The correlation coefficients in the linear part of the curves, measured with the ISFET, CWE and ISE, were 0.999, 1.000 and 0.999 , respectively.

\subsection{Response time measurements}

A FIA set-up was used to measure the response times of the ISFET, CWE and ISE. We determined the $t_{90}$ response time, as recommended by IUPAC [26]. $t_{90}$ is defined as the time required to reach $90 \%$ of the signal steady-state value. We injected rectangular concentration pulses of $10^{-3} \mathrm{M}$ fumaric acid, using a $500 \mu \mathrm{l}$ injection loop (pulse time of $15 \mathrm{~s}$ ). Fig. 4 gives the response time $t_{90}$ as a function of the flow-rate for the three sensors. It reveals that the response time increases when the flow-rate is lowered. This is caused by the increased thickness of the diffusion layer near the membrane [14]. The three types of potentiometric detectors all show acceptable response times for a conventional LC system. However, both the CWE and ISFET type sensors have a faster response time than

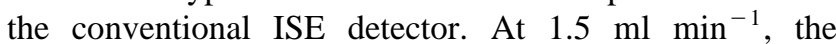

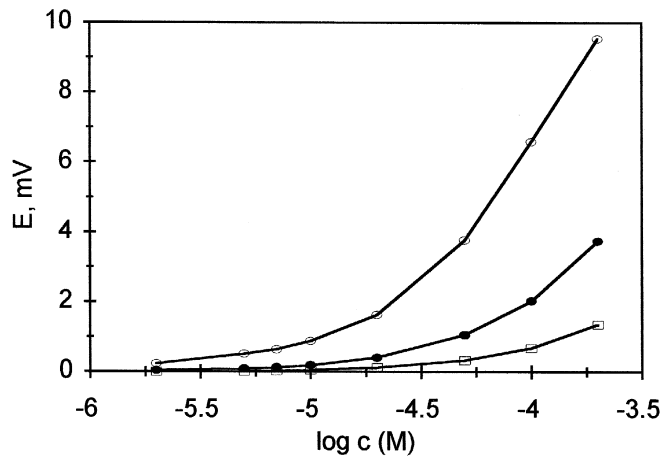

Fig. 3. Calibration curves of fumaric acid measured at an ISFET $(O)$, a CWE (○) and an ISE ( $\square$ ) in anion-exchange chromatography. Conditions as in Fig. 1.

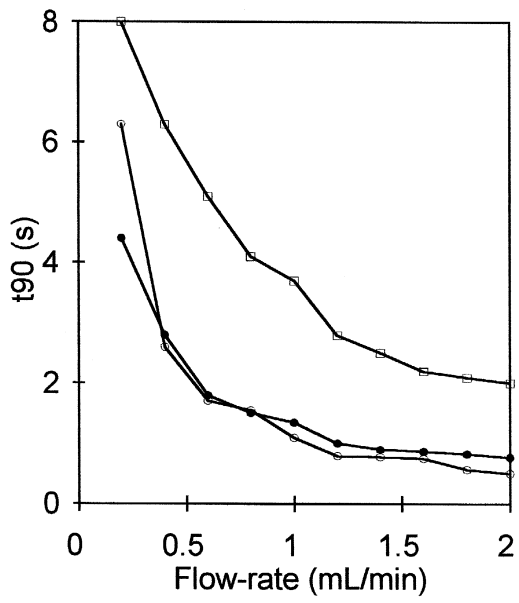

Fig. 4. Response time as a function of flow-rate for the ISFET $(\bigcirc)$, CWE (O) and ISE ( $\square$ ). Measurements were done in a FIA set-up using the chromatographic conditions as in Fig. 1. Concentration plugs of $10^{-3} \mathrm{M}$ fumaric acid were injected.

response times of the ISFET and CWE were both around 1 $\mathrm{s}$, whereas for the ISE this value was bigger than $2 \mathrm{~s}$.
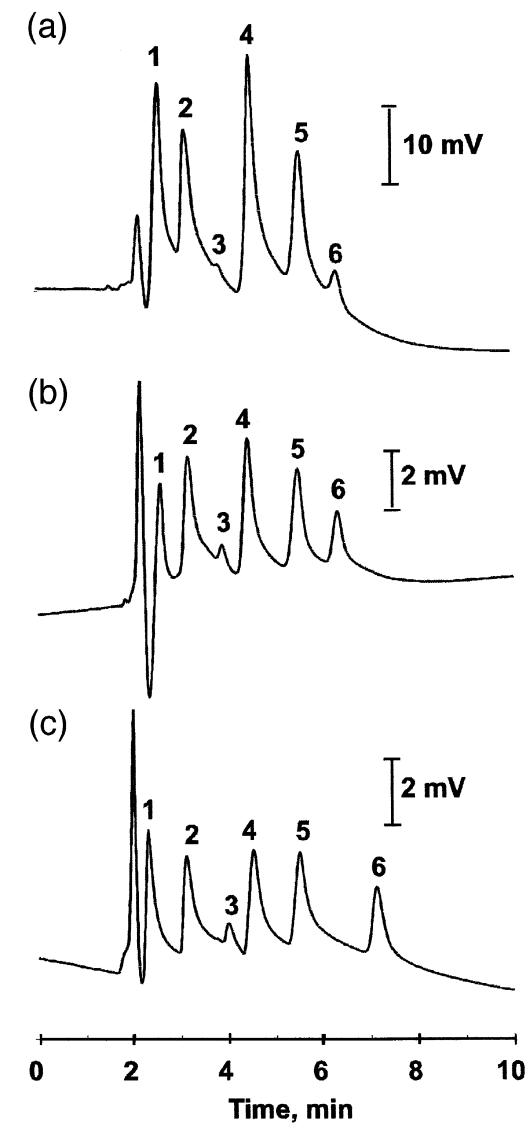

Fig. 5. Chromatograms of a mixture of six carboxylic acids obtained using three different potentiometric sensor types: (a) ISFET, (b) CWE and (c) ISE. Column: Merck Lichrospher RP-8, $125 \times 4 \mathrm{~mm}$ i.d. Flow-rate:

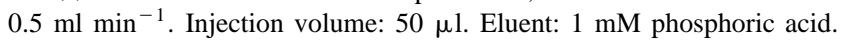
Injected concentration: $10^{-4}$ M. Peak identification: (1) tartaric acid, (2) malic acid, (3) lactic acid, (4) citric acid, (5) fumaric acid and (6) succinic acid. 


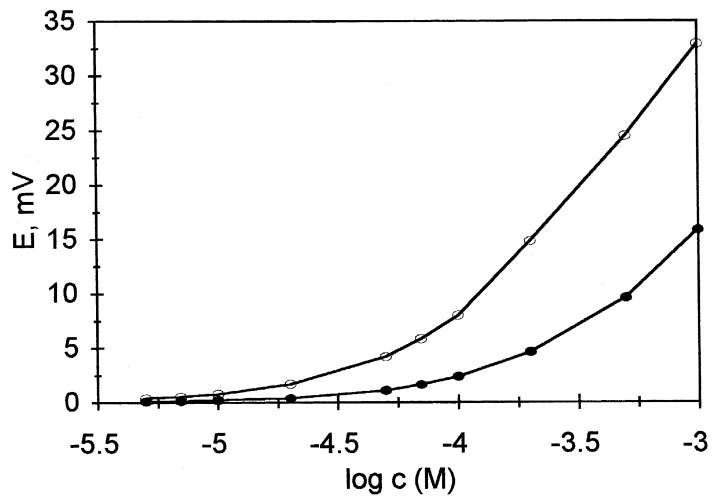

Fig. 6. Calibration curves of fumaric acid measured at an ISFET $(\bigcirc)$ and a CWE ( ) in reversed-phase chromatography. Conditions as in Fig. 4.

\subsection{Reversed-phase chromatography}

In reversed-phase chromatography, eluents of low $\mathrm{pH}$ are required to separate organic acids. These eluents suppress the ionisation of the acids. In a previous study [16], it was shown that a $1 \mathrm{mM}$ phosphoric acid eluent allowed a sensitive potentiometric detection of organic acids in this system, using a conducting oligomer electrode. Chromatograms were measured under the same conditions for the three sensor types (see Fig. 5). The mixture of organic acids contained tartaric, malic, lactic, citric, fumaric and succinic acid.

The detection limit (signal/noise ratio $=3$ ) for fumaric acid was $5 \times 10^{-6} \mathrm{M}$ for the three sensors. This corresponds to an injected amount of 250 pmol. The detection limit measured with an UV detector for this acid is much lower, due to the high absorption of its conjugated double bond. If we consider the other carboxylic acids, which lack a chromophoric group, the sensors offer a sensitivity that is comparable to that of UV detection at $210 \mathrm{~nm}$ [16].

Calibration curves for fumaric acid were measured with the ISFET and CWE. They are presented in Fig. 6. Both curves showed linearity up to injected concentrations of $2 \times 10^{-4} \mathrm{M}$.

The correlation coefficients were 0.997 for the ISFET and 1.000 for the CWE.

\section{Conclusions}

This study demonstrates the possibility of using an ISFET-based sensor for the determination of organic acids in two different LC systems (anion-exchange and reversed phase chromatography). The results show that the ISFET can be used as a simple and inexpensive alternative for the classical detectors (suppressed conductivity and low-wavelength UV). It was found that the detection limits obtained with the ISFET were comparable to those obtained with the CWE or the conventional type of ISE (of the order of 100 pmol in the anion-exchange system). The response times of the ISFET- and CWE-based systems were also comparable and somewhat superior to the conventional ISE type configuration. Similar calibration curves were also obtained for the CWE and ISFET based systems, showing a linear relationship in the lower part of the curve. Both the ISFET and the CWE systems are interesting detection devices for use in miniaturised systems of analysis such as micro-Total Analysis System ( $\mu$ TAS). The CWE system is very simple, and more robust (see Ref. [24]) than the ISFET system where poor adhesion of the coating to the gate part was sometimes observed - this should be improved in future work. The ISFET system allows transport of a low impedance signal to the data station enabling a high degree of miniaturisation and integration in "lab on a chip" or $\mu$ TAS systems [27].

\section{Acknowledgements}

This research has been partially supported by the Technology Foundation STW, applied science division of NWO, Project Biomass no. DST 66.4351. The authors kindly acknowledge Prof. P. Bergveld and J. Bomer of the Biosensors group, MESA +, University of Twente for delivering the ISFETs. L. Nagels thanks the FWO for financial support.

\section{References}

[1] M. Olalla Herrera, H. López Garciá, M. Villalón Mir, M.C. López Martínez, Determination by high-performance liquid chromatography of organic acids in Spanish rosé wines from the alpujarra-contraviesa region of Granada, J. Liq. Chromatogr. 16 (1993) 31013112.

[2] M.W. Dong, HPLC analysis of organic acids in juice and wine using resin and reversed-phase columns, LC-GC 16 (1998) 1092-1097.

[3] P. Hajós, L. Nagy, Retention behaviours and separation of carboxylic acids by ion-exchange chromatography, J. Chromatogr., B 717 (1998) 27-38.

[4] P.A.W. Van Hees, J. Dahlén, U.S. Lundström, H. Borén, B. Allard, Determination of low molecular weight organic acids in soil solution by HPLC, Talanta 48 (1999) 173-179.

[5] B.K. Glod, P.W. Alexander, P.R. Haddad, Z.L. Chen, Potentiometric detection using a metallic copper electrode in reversed-phase and ion-exclusion chromatography with eluents containing ion-interaction reagents, J. Chromatogr., A 699 (1995) 31-37.

[6] C.W. Klampfl, W. Buchberger, Determination of low-molecularmass organic acids by capillary zone electrophoresis, TrAC 16 (1997) 221-229.

[7] A. Manz, W. Simon, Potentiometric detector for fast high-performance open-tubular column liquid chromatography, Anal. Chem. 59 (1987) 74-79.

[8] A. Nann, I. Silvestri, W. Simon, Quantitative analysis in capillary zone electrophoresis using ion-selective microelectrodes as on-column detectors, Anal. Chem. 65 (1993) 1662-1667.

[9] Z.L. Chen, D.B. Hibbert, Simultaneous amperometric and potentiometric detection of sugars, polyols and carboxylic acids in flow systems using copper wire electrodes, J. Chromatogr. A 766 (1997) $27-33$. 
[10] Z.L. Chen, P.W. Alexander, P.R. Haddad, Liquid chromatography of carboxylic acids using potentiometric detection with a tungsten oxide electrode, Anal. Chim. Acta 338 (1997) 41-49.

[11] T. Kappes, P.C. Hauser, Potentiometric detection in capillary electrophoresis with a metallic copper electrode, Anal. Chim. Acta 354 (1997) 129-134.

[12] P. Schnierle, T. Kappes, P.C. Hauser, Capillary electrophoretic determination of different classes of organic ions by potentiometric detection with coated-wire ion-selective electrodes, Anal. Chem. 70 (1998) 3585-3589.

[13] I. Isildak, Potentiometric detection of monovalent anions separated by ion chromatography using all solid-state contact PVC matrix membrane electrode, Chromatographia 49 (1999) 338-342.

[14] B.L. De Backer, L.J. Nagels, Potentiometric detection of organic acids in ion-exclusion chromatography using different types of liquid-membrane electrodes, Anal. Chim. Acta 290 (1994) 259-267.

[15] B.L. De Backer, L.J. Nagels, Potentiometric detection for capillary electrophoresis: determination of organic acids, Anal. Chem. 68 (1996) 4441-4445.

[16] I. Poels, L.J. Nagels, G. Verreyt, H.J. Geise, Potentiometric detection of organic acids in liquid chromatography using conducting oligomer electrodes, Anal. Chim. Acta. 370 (1998) 105-113.

[17] I. Poels, L.J. Nagels, Conducting polymer and oligomer micro-electrodes for the potentiometric detection of anions in capillary electrophoresis, Anal. Chim. Acta 401 (1999) 21-27.

[18] C. Jiménez, I. Marqués, J. Bartrolí, Continuous-flow system for on-line water monitoring using back-side contact ISFET-based sensors, Anal. Chem. 68 (1996) 3801-3807.

[19] S. Alegret, J. Bartrolí, C. Jimenez-Jorquera, M. del Valle, Flowthrough $\mathrm{pH}-\mathrm{ISFET}+$ reference-ISE as integrated detector in automated FIA determinations, Sens Actuators, B 7 (1992) 555-560.
[20] K. Watanabe, K. Tohda, H. Sugimoto, F. Eitoku, H. Inoue, K. Suzuki, S. Nakamura, Ion-sensitive field effect transistor as a monovalent cation detector for ion chromatography and its application to the measurement of $\mathrm{Na}^{+}$and $\mathrm{K}^{+}$concentrations in serum, J. Chromatogr. 566 (1991) 109-116.

[21] P. Bergveld, A. Sibbald, Analytical and biomedical application of ion-selective field effect transistors, in: Comprehensive Analytical Chemistry vol. XXIII Elsevier, Amsterdam, 1988, pp. 75-100.

[22] L.J. Nagels, J.M. Kauffmann, C. Dewaele, F. Parmentier, Convection/diffusion- and diffusion-controlled rapid-scan voltammetry in liquid chromatographic systems with a large-volume wall-jet detector, Anal. Chim. Acta 234 (1990) 75-81.

[23] D. Wegmann, H. Weiss, D. Ammann, W.E. Morf, E. Pretsch, K. Sugahara, W. Simon, Anion-selective liquid membrane electrodes based on lipophilic quaternary ammonium compounds, Mikrochim. Acta III (1984) 1-16.

[24] S. Picioreanu, I. Poels, J. Frank, J.C. van Dam, G.W.K. van Dedem, L.J. Nagels, Potentiometric detection of carboxylic acids, phosphate esters and nucleotides in liquid chromatography using anion-selective coated-wire electrodes, Anal. Chem. 72 (2000) 2029-2034.

[25] M. Bhattacharya, L. Fuhrman, A. Ingram, K.W. Nickerson, T. Conway, Single-run separation and detection of multiple metabolic intermediates by anion-exchange high-performance liquid chromatography and application to cell pool extracts prepared from Escherichia coli, Anal. Biochem. 232 (1995) 98-106.

[26] G.G. Guilbault, Recommendations for publishing manuscripts on ion-selective electrodes, IUPAC Inf. Bull. 1 (1978) 69-74.

[27] M. Niu, X. Ding, X. Tao, W. Lin, in: Development of a novel micro FIA-ISFET integrated sensor, Proceedings of the 1995 IEEE Region 10 Annual International Conference on Microelectronics and VLSI, TENCON, 1995, pp. 183-186. 